\title{
Upper limits on the solar-neutron flux at the Yangbajing neutron monitor from BATSE-detected solar flares
}

\author{
H. Tsuchiya ${ }^{1}$, H. Miyasaka ${ }^{2}$, E. Takahashi ${ }^{1}$, S. Shimoda ${ }^{1}$, Y. Yamada ${ }^{1}$, I. Kondo ${ }^{1}$, K. Makishima ${ }^{1,3}$,

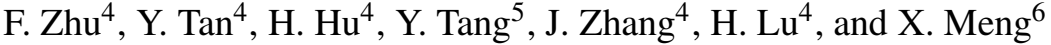 \\ 1 RIKEN, 2-1, Hirosawa, Wako, Staitama 351-0198, Japan \\ e-mail: harufumi@crab.riken.jp \\ 2 Caltech, Pasadena, CA 91125, USA \\ 3 Department of Physics, University of Tokyo, 7-3-1, Hongo, Bunkyo-ku, Tokyo 113-0033, Japan \\ 4 Institute of High Energy Physics, Beijing 100049, PR China \\ 5 China Meteorological Administration, Beijing 100049, PR China \\ 6 Tibet University, Lhasa 850000, PR China
}

Received 2 November 2006 / Accepted 12 March 2007

\section{ABSTRACT}

\begin{abstract}
Aims. The purpose of this work is to search the data from Yangbajing neutron monitor obtained between 1998 October and 2000 June for the solar neutrons associated with solar flares.

Methods. Using the onset times of 166 BATSE-detected flares with the GOES peak flux (1-8 $\AA$ ) higher than $1.0 \times 10^{-5} \mathrm{Wm}^{-2}$, we prepared a light curve of the Yangbajing neutron monitor for each flare, spanning $\pm 1.5 \mathrm{~h}$ from the BATSE onset time. Based on the light curves, a systematic search for solar neutrons in energies above $100 \mathrm{MeV}$ from the 166 flares was performed. No statistically significant signals due to solar neutrons were found. Therefore, we put upper limits on the $\geq 100 \mathrm{MeV}$ solar-neutron flux for 18 events consisting of $2 \mathrm{X}$ and $16 \mathrm{M}$ class flares. The calculation assumed a power-law shaped neutron energy spectrum and three types of neutron emission profiles at the Sun.

Results. Compared with the other positive neutron detections associated with X-class flares, typical 95\% confidence level upper limits for the two X-class flares are found to be comparable to the lowest and second lowest neutron fluxes at the top of the atmosphere. In addition, the upper limits for M-class flares scatter in the range of $10^{-2}$ to 1 neutrons $\mathrm{cm}^{-2} \mathrm{~s}^{-1}$. This provides the first upper limits on the solar-neutron flux from M-class solar flares, using space observatories, as well as ground-based neutron monitors.
\end{abstract}

Key words. Sun: flares - Sun: particle emission - Sun: X-rays, gamma rays

\section{Introduction}

Solar neutrons, as well as nuclear gamma rays, have been widely used as a good probe of the still unclear fundamental mechanisms of ion acceleration associated with solar flares. These neutral secondaries emanate from deep in the chromosphere via nuclear processes between accelerated ions and the solar ambient plasma.

Early quantitative studies (Hess 1962; Lingenfelter et al. 1965a,b; Lingenfelter 1969) predicted that large solar flares would produce a measurable flux of solar neutrons at the Earth, $\sim 10 \mathrm{~cm}^{-2} \mathrm{~s}^{-1}$ in energies above $10 \mathrm{MeV}$. Before the solar cycle 21, various balloon-borne experiments attempted to detect the predicted neutrons originating from solar flares (Apparao et al. 1966; Daniel et al. 1967; Hess \& Kaifer 1967; Daniel et al. 1969; Forrest \& Chupp 1969), but none gave convincing results.

In the solar cycle 21 and 22, the predicted solar neutrons have actually been detected from several X-class solar flares by satellite-borne or ground-based detectors (Chupp et al. 1982, 1987; Muraki et al. 1992; Debrunner et al. 1993; Struminsky et al. 1994). The first clear detection was achieved with the Gamma-Ray Spectrometer (GRS) onboard the Solar Maximum Mission (SMM), on the occasion of an X2.6 solar flare on 1980 June 21 (Chupp et al. 1982). On the other hand, all groundbased positive detections were associated with more energetic solar flares, with the GOES class larger than X8. While these successful detections indeed demonstrate the production of solar neutrons in solar flares, it was thought that the neutron detection from medium X-class flares would be difficult with groundbased observations.

Surprisingly, solar neutrons were successfully detected from an X2.3 solar flare on 2000 November 24, near the maximum of the solar cycle 23 , by a neutron monitor installed at Mt. Chacaltaya in Bolivia (Watanabe et al. 2003). The Bolivian neutron monitor is situated at an altitude of $5250 \mathrm{~m}$ and has an area of $13 \mathrm{~m}^{2}$. This observation therefore led to groundbased detectors on very high mountains being able to detect solar neutrons even from medium X-class solar flares, although the 2000 November 24 event might be exceptional.

Because of the small $(\sim 10)$ number of positive detections of solar neutrons, it is not clear at present how the arrival flux of solar neutrons depends on the flare intensity. Furthermore, it is unlikely that the neutron detections will increase significantly in number in the near future. Therefore, we may even utilize upper limits on the solar-neutron flux for a much larger sample of solar flares. These upper limits at the Earth are expected to constrain some key parameters of the underlying ion acceleration process in solar flares, including the total number, the energy content, and pitch angle distributions of the accelerated ions. In the present paper, we hence analyze the data from the Yangbajing neutron monitor for any signals associated with 
$166 \mathrm{M}$ - and X-class flares, detected with the BATSE (Burst And Transient Source Experiment) on board Compton Gamma Ray Observatory (CGRO) over the period of 1998 October through 2000 June.

In Sect. 2 we give an overview of the Yangbajing neutron monitor, and describe its performance using Monte Carlo simulations in Sect. 3. Section 4 presents our methods used in this analysis. The derived results are given in Sect. 5, together with a brief discussion.

\section{The Yangbajing neutron monitor}

The Yangbajing neutron monitor (NM) has been operated at Yangbajing $\left(90.522 \mathrm{E}, 30^{\circ} .102 \mathrm{~N}\right)$ in Tibet, China, since 1998 October (Kohno et al. 1999; Miyasaka et al. 2001). Its high altitude, $4300 \mathrm{~m}$ above sea level, provides a much reduced air mass, $606 \mathrm{~g} \mathrm{~cm}^{-2}$. It consists of $28 \mathrm{NM64}$ type detectors (Carmichael 1964; Stoker et al. 2000), attaining a total area of $31.7 \mathrm{~m}^{2}$ which is the largest one among the world-wide NM network at present. Another advantage of the Yangbajing NM is that it has the highest geomagnetic cutoff rigidity, $14 \mathrm{GV}$, among NMs in the world, thanks to geomagnetic conditions. These conditions make the Yangbajing NM one of the most sensitive detectors for solar neutrons.

\section{Performance of the Yangbajing neutron monitor}

The data acquisition system of the Yangbajing NM records the event number of each counter every second. The counting rate is typically $100 \mathrm{~Hz}$ per counter, so the total rate becomes around $2.8 \mathrm{kHz}$. In order to examine whether this value is reasonable, we calculated the expected counting rate of the Yangbajing NM by means of a full Monte Carlo (MC) simulation in the atmosphere called Cosmos uv6.35 (Kasahara 2003). Primary cosmic-ray particles were sampled from the energy spectrum constructed from direct observations in the energy range from $10 \mathrm{GeV}$ to $100 \mathrm{TeV}$ (Asakimori et al. 1998; Sanuki et al. 2000; Apanasenko et al. 2001; Sanuki et al. 2001). An error of $\pm 20 \%$ was assigned to the absolute flux of primary particles in lower energies, whereas its uncertainty was set to be $\pm 50 \%$ at around $100 \mathrm{TeV}$. These primary particles were thrown isotropically within the zenith angle $0^{\circ}$ to $60^{\circ}$ on top of the atmosphere, and then fluxes of various secondary particles at the altitude of Yangbajing were evaluated. The flux of species of each secondary particle was finally multiplied by the detection efficiency of the NM64 detector calculated by Clem \& Droman (2000), which in turn was confirmed via comparison with an accelerator experiment using 100-400 MeV neutron beams (Shibata et al. 2001).

As a consequence of the calculation, the expected counting rate of the Yangbajing NM was estimated as $3.4 \mathrm{kHz}$. This agrees with the experimental counting rate of $2.8 \mathrm{kHz}$ within the error of the absolute flux of primary particles. In addition, the MC simulation revealed how various secondary particle species contribute to the total counting rate. The secondary neutrons were found to dominate the counting rate with a typical fraction of $83 \%$. The next dominant contribution comes from protons, with $15 \%$ to the total counting rate. The remaining marginal $2 \%$ is mainly due to secondary negative muons that are captured in the lead blocks through a reaction of $\mu^{-}+\mathrm{p} \rightarrow \mathrm{n}+v_{\mu}$. This result ensures that $98 \%$ of the counting rate is dominated by secondary nucleons, as expected.

For a further analysis, we also derived an attenuation factor of solar neutrons in the atmosphere. The attenuation of solar neutrons is caused by their collisions with atmospheric nuclei during

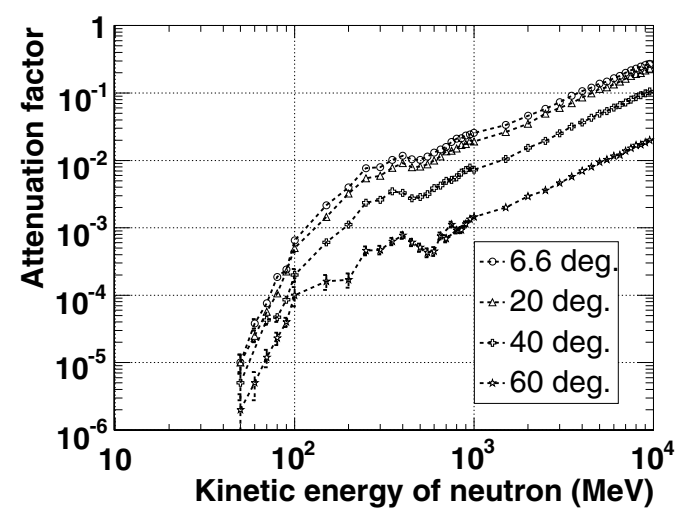

Fig. 1. The atmospheric attenuation factor of solar neutrons, calculated via Monte-Carlo simulations assuming different incident zenith angles, and shown as a function of kinetic energy of neutrons measured at the top of the Earth atmosphere.

their propagation to the observers, depending on their kinetic energies and incident angles. This is illustrated in Fig. 1, which was obtained using MC simulations of CORSIKA 6.500 (Heck et al. 1998) embedding GHEISHA (Fesefeldt 1985; Cassell \& Bower $2002)$ as a low-energy $(<80 \mathrm{GeV} / \mathrm{n})$ hadronic interaction model. Thus, low-energy solar neutrons are strongly reduced in flux, particularly when the incident zenith angle is large. For example, solar neutrons with a kinetic energy of $100 \mathrm{MeV}$ and an incident angle of $60^{\circ}$ suffer heavy attenuation by four orders of magnitude. Even with such a heavy attenuation, we expect successful detections of solar neutrons with the Yangbajing NM, if the arriving neutron flux exceeds $\sim 1 \mathrm{~cm}^{-2} \mathrm{~s}^{-1}$ as found in some past solar flares with the GOES class above X10, and if each event lasts typically $\sim 10 \mathrm{~min}$.

\section{Analysis}

\subsection{Flare sample definition}

Since the beginning of its operation on 1991 April 19 through the re-entry to the atmosphere on 2000 June 4, the BATSE aboard CGRO observed more than 7000 solar flares in the hard X-ray range above $\sim 25 \mathrm{keV}$; the flare list is publicly available ${ }^{1}$. Among them, 1205 events were detected over a period of 1998 October and 2000 June, i.e., overlapping with the Yangbajing NM operation, with the GOES peak flux $\left(F_{\mathrm{GOES}}\right)$ higher than $1.0 \times$ $10^{-6} \mathrm{Wm}^{-2}$, which corresponds to the GOES class of $\mathrm{C} 1$. The flare number reduces to 1013 when we exclude those events where the Yangbajing NM did not operate due to maintenance or system troubles. The 1013 events, composed of 847 C-class, $159 \mathrm{M}$-class, and $7 \mathrm{X}$-class flares, constitute our "very preliminary sample", which is further divided into two subsets. One subset is called "preliminary sample", consisting of the 166 flares with the GOES class larger than M1. The other is "subpreliminary sample" with the $847 \mathrm{C}$-class flares.

As indicated by Fig. 1, the zenith angle of the Sun at Yangbajing, $\theta_{\mathrm{SUN}}$, must be small in order for the Yangbajing NM to detect neutron signals. Therefore, we arranged the 1013 "very preliminary" sample events, in Fig. 2 , on the plane of $\cos \left(\theta_{\text {SUN }}\right)$ calculated at the flare onset vs. $F_{\mathrm{GOES}}$. Based on this plot, we have selected 18 flares for our "final sample", with criteria that $\theta_{\mathrm{SUN}}$ is smaller than $60^{\circ}$ and $F_{\mathrm{GOES}}$ is higher than $1.0 \times$ $10^{-5} \mathrm{Wm}^{-2}$. These flares are summarized in Table 1 .

\footnotetext{
1 ftp://umbra.nascom.nasa.gov/pub/batse/events/
} 
Table 1. The 18 solar flares in the final sample.

\begin{tabular}{|c|c|c|c|c|c|c|c|c|}
\hline $\begin{array}{l}\text { Date } \\
\text { (YYMMDD) }\end{array}$ & Class/optical importance $^{a}$ & Position & $\begin{array}{l}t_{\mathrm{S}}^{b} \\
\text { (UT) }\end{array}$ & $\begin{array}{l}t_{\mathrm{P}}^{c} \\
\text { (UT) }\end{array}$ & $\begin{array}{l}\Delta t_{\mathrm{b}}{ }^{d} \\
(\mathrm{~s})\end{array}$ & $\begin{array}{c}N_{\mathrm{P}}{ }^{e} \\
\left(2000 \mathrm{~cm}^{-2} \mathrm{~s}^{-1}\right)\end{array}$ & $N_{\mathrm{T}}^{f}$ & $\begin{array}{l}\theta_{\text {SUN }^{g}} \\
(\mathrm{deg})\end{array}$ \\
\hline 981112 & $\mathrm{M} 1.0 / 1 \mathrm{~N}$ & N21W34 & $5^{\mathrm{h}} 26^{\mathrm{m}} 23^{\mathrm{s}}$ & $5^{\mathrm{h}} 27^{\mathrm{m}} 6^{\mathrm{s}}$ & 124 & 45542 & 777267 & 48 \\
\hline 981122 & X3.7/1N & S27W82 & $6^{\mathrm{h}} 33^{\mathrm{m}} 36^{\mathrm{s}}$ & $6^{\mathrm{h}} 39^{\mathrm{m}} 17^{\mathrm{s}}$ & 901 & 1130000 & 168000000 & 51 \\
\hline 981128 & $\mathrm{X} 3.3 / 3 \mathrm{~N}$ & N17E32 & $5^{\mathrm{h}} 31^{\mathrm{m}} 36^{\mathrm{s}}$ & $5^{\mathrm{h}} 40^{\mathrm{m}} 46^{\mathrm{s}}$ & 2834 & 670736 & 120056656 & 52 \\
\hline 981217 & $\mathrm{M} 3.2 / 1 \mathrm{~N}$ & S27W46 & $7^{\mathrm{h}} 41^{\mathrm{m}} 41^{\mathrm{s}}$ & $7^{\mathrm{h}} 43^{\mathrm{m}} 16^{\mathrm{s}}$ & 136 & 120179 & 3418773 & 59 \\
\hline 990402 & M1.1/ & & $8^{\mathrm{h}} 9^{\mathrm{m}} 14^{\mathrm{s}}$ & $8^{\mathrm{h}} 11^{\mathrm{m}} 48^{\mathrm{s}}$ & 453 & 5960 & 727034 & 38 \\
\hline 990404 & $\mathrm{M} 5.4 / 1 \mathrm{~F}$ & N18E72 & $5^{\mathrm{h}} 24^{\mathrm{m}} 57^{\mathrm{s}}$ & $5^{\mathrm{h}} 25^{\mathrm{m}} 9^{\mathrm{s}}$ & 114 & 1085 & 63400 & 26 \\
\hline 990503 & $\mathrm{M} 4.4 / 2 \mathrm{~N}$ & N15E32 & $5^{\mathrm{h}} 39^{\mathrm{m}} 55^{\mathrm{s}}$ & $5^{\mathrm{h}} 47^{\mathrm{m}} 52^{\mathrm{s}}$ & 2523 & 26942 & 6403993 & 14 \\
\hline 990510 & $\mathrm{M} 2.5 / 2 \mathrm{~N}$ & N16E19 & $5^{\mathrm{h}} 24^{\mathrm{m}} 35^{\mathrm{s}}$ & $5^{\mathrm{h}} 26^{\mathrm{m}} 45^{\mathrm{s}}$ & 561 & 13420 & 955186 & 14 \\
\hline 990529 & M1.6/ & & $3^{\mathrm{h}} 6^{\mathrm{m}} 43^{\mathrm{s}}$ & $3^{\mathrm{h}} 8^{\mathrm{m}} 50^{\mathrm{s}}$ & 885 & 17123 & 2420462 & 39 \\
\hline 990604 & M3.9/2B & N17W69 & $6^{\mathrm{h}} 53^{\mathrm{m}} 29^{\mathrm{s}}$ & $6^{\mathrm{h}} 59^{\mathrm{m}} 58^{\mathrm{s}}$ & 1076 & 490858 & 44716592 & 14 \\
\hline 990724 & M3.3/SF & S28E78 & $7^{\mathrm{h}} 49^{\mathrm{m}} 54^{\mathrm{s}}$ & $8^{\mathrm{h}} 1^{\mathrm{m}} 24^{\mathrm{s}}$ & 849 & 19173 & 1727533 & 25 \\
\hline 990725 & $\mathrm{M} 1.0 / 1 \mathrm{~F}$ & S26E58 & $4^{\mathrm{h}} 22^{\mathrm{m}} 17^{\mathrm{s}}$ & $4^{\mathrm{h}} 24^{\mathrm{m}} 1^{\mathrm{s}}$ & 133 & 806 & 13297 & 27 \\
\hline $991116 a$ & $\mathrm{M} 1.8 / \mathrm{SF}$ & N18E43 & $4^{\mathrm{h}} 48^{\mathrm{m}} 0^{\mathrm{s}}$ & $4^{\mathrm{h}} 48^{\mathrm{m}} 52^{\mathrm{s}}$ & 105 & 651 & 32910 & 50 \\
\hline $991116 b$ & $\mathrm{M} 2.3 / 1 \mathrm{~N}$ & S14E10 & $6^{\mathrm{h}} 3^{\mathrm{m}} 38^{\mathrm{s}}$ & $6^{\mathrm{h}} 7^{\mathrm{m}} 31^{\mathrm{s}}$ & 491 & 21992 & 2695715 & 49 \\
\hline 991126 & M1.9/2B & S19E58 & $3^{\mathrm{h}} 52^{\mathrm{m}} 56^{\mathrm{s}}$ & $3^{\mathrm{h}} 55^{\mathrm{m}} 8^{\mathrm{s}}$ & 665 & 18033 & 1763834 & 58 \\
\hline 000408 & $\mathrm{M} 2.0 / 1 \mathrm{~B}$ & S15E26 & $2^{\mathrm{h}} 38^{\mathrm{m}} 13^{\mathrm{s}}$ & $2^{\mathrm{h}} 38^{\mathrm{m}} 38^{\mathrm{s}}$ & 523 & 189878 & 7243484 & 52 \\
\hline 000504 & $\mathrm{M} 2.8 / 1 \mathrm{~N}$ & S14W90 & $4^{\mathrm{h}} 34^{\mathrm{m}} 33^{\mathrm{s}}$ & $4^{\mathrm{h}} 37^{\mathrm{m}} 28^{\mathrm{s}}$ & 744 & 34745 & 3111922 & 23 \\
\hline 000515 & M4.4/ & & $8^{\mathrm{h}} 33^{\mathrm{m}} 43^{\mathrm{s}}$ & $8^{\mathrm{h}} 42^{\mathrm{m}} 46^{\mathrm{s}}$ & 3566 & 38435 & 18834356 & 33 \\
\hline
\end{tabular}

${ }^{a}$ The optical importance of some solar flares is unavailable from the GOES catalog. ${ }^{b}$ BATSE start time. ${ }^{c}$ BATSE peak time. ${ }^{d}$ BATSE duration. ${ }^{e}$ BATSE peak rate. ${ }^{f}$ BATSE total counts. ${ }^{g}$ A zenith angle of the Sun for individual flares at the onset time.

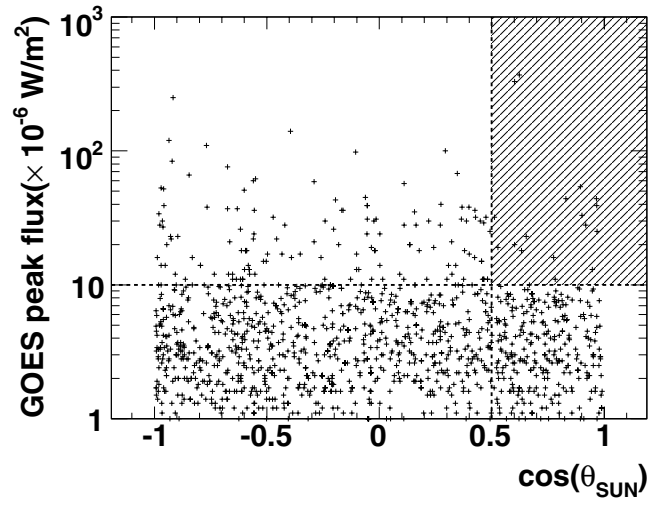

Fig. 2. The cosine of the zenith angle of the Sun $\left(\theta_{\text {SUN }}\right)$ at Yangbajing at the onset time of each flare in our very preliminary sample, plotted against their GOES peak flux in units of $10^{-6} \mathrm{~W} \mathrm{~m}^{-2}$. The vertical dashed line indicates $\theta_{\text {SUN }}=60^{\circ}$, while the horizontal one represents the GOES flux of $1 \times 10^{-5} \mathrm{~W} \mathrm{~m}^{-2}$, or the GOES class of M1. The 18 events in the hatched area constitute our final sample.

\subsection{NM light curves}

Our basic strategy is to search the NM count-rate histories (or "light curves") for possible excess counts associated with solar flares. For each flare in our preliminary sample, we therefore prepared a light curve from the Yangbajing NM, spanning $\pm 1.5 \mathrm{~h}$ from the flare onset time, $t_{\mathrm{S}}$, determined by the BATSE data. This time interval is considered long enough to cover each flare and to estimate background count levels. We binned the light curves into 5 min per bin and corrected them for atmospheric pressure changes that cause variations in the attenuation factor typically by $\pm 2 \%$. Figure 3 shows four particular examples of such light curves out of the 18 final-sample flares; these are two X-class flares, 981122 and 981128, and two M-class ones, 990604 and 000408 , with the last two showing the highest and the second highest BATSE peak rates among M-class flares in our final sample (Table 1). Thus, no excess counts due to solar neutrons are apparently observed.

In order to more quantitatively constrain neutron counts associated with each flare, we need to define an "ON time window" for each flare, i.e., a time interval when solar neutrons might indeed arrive at the Yangbajing NM, and use the remaining two time intervals (before and after the flare) to estimate the background. For this purpose, we must in turn assume a time profile of the solar-neutron production at the Sun in each flare, as well as the maximum and minimum kinetic energies of the produced solar neutrons. Then the "ON time window"opens at the arrival of the most energetic neutrons ejected at the beginning of the neutron emission at the Sun and closes at that of the least energetic ones ejected at the end of the production interval. In this work, we postulate that the maximum and minimum kinetic energies of solar neutrons are $10 \mathrm{GeV}$ and $100 \mathrm{MeV}$, respectively.

The following three neutron-emission time profiles are employed; (1) $\delta$-emission, (2) continuous-emission, and (3) Gaussian-emission. the $\delta$-emission simply means that solar neutrons are emitted from the Sun instantaneously at the BATSE HXR emission peak, while the continuous-emission profile assumes that neutrons are continuously and constantly radiated from the Sun throughout the BATSE HXR emission. Here for the purpose of introducing the mathematical forms of each emission profile, we define "re-normalizing" time $t$ as $T-t_{\mathrm{S}}$, where $T$ and $t_{\mathrm{S}}$ show the normal time measured in UT and the onset time of the BATSE emission, respectively. With the renormalizing time $t$ and above assumptions, the neutron emission profile $v(t)$ takes either of the following forms; $v(t)=1$ only at $t=t_{\mathrm{P}}-t_{\mathrm{S}}$ and otherwise 0 if the $\delta$-emission is adopted, $v(t)=1$ for $t=0$ to $\Delta t_{\mathrm{b}}$ and otherwise 0 if the continuous-emission profile is used. Here, $t_{\mathrm{P}}$ is the peak time of the BATSE emission, and $\Delta t_{\mathrm{b}}$ is the BATSE flare duration. Hereafter we define $T_{\mathrm{PS}}=t_{\mathrm{P}}-t_{\mathrm{S}}$. Both these cases have been considered in some past studies (Chupp et al. 1982; Muraki et al. 1992; Shibata 1994; Watanabe et al. 2003; Bieber et al. 2005). 

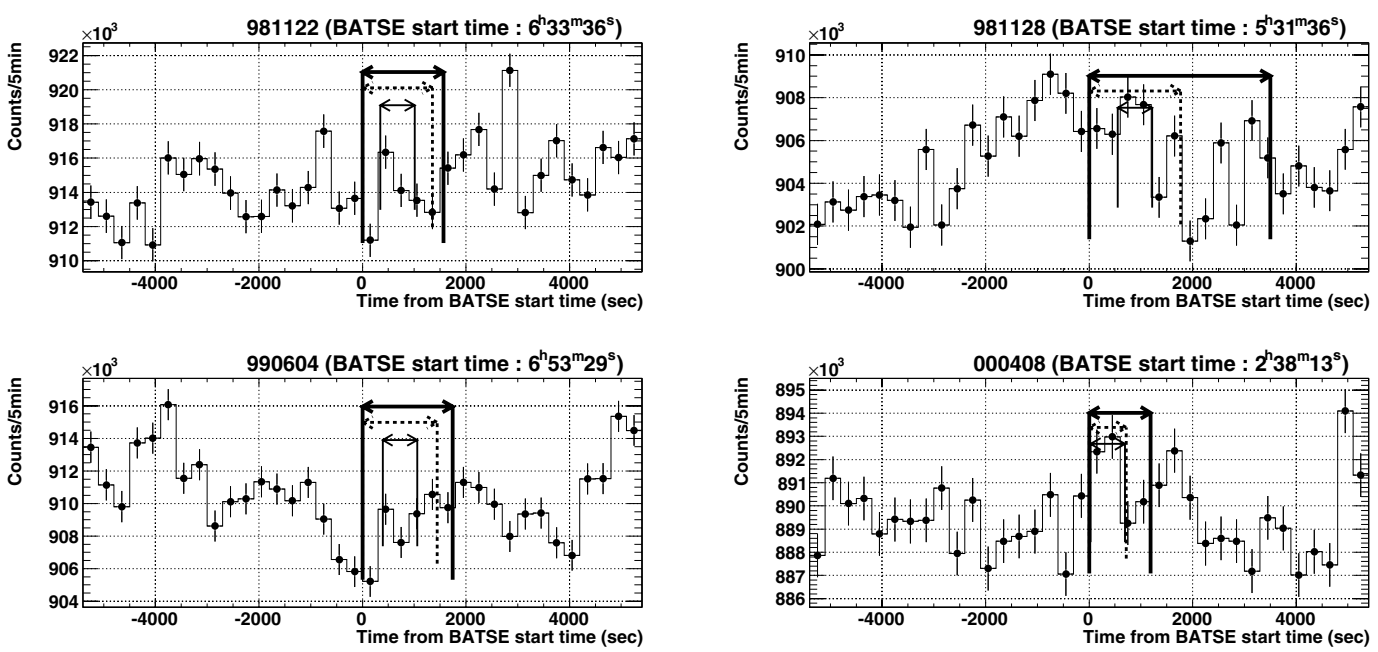

Fig. 3. The 5-min counting-rate histories of the Yangbajing NM for two X-class and two M-class flares. Left-upper and right-upper panels show light curves for the X3.7 (981122) and X3.3 (981128) flares, respectively, while left-lower and right-lower panels indicate those for the M3.9 (990604) and M2.0 (000408) flares, respectively. Abscissa in all panels show the time measured from $t_{\mathrm{s}}$, ranging over $\pm 5400 \mathrm{~s}$, where zero corresponds to the BATSE start time (Table 1). The intervals of individual ON time windows specified by the $\delta$-, continuous-, and Gaussianemission profiles are indicated by horizontal thin, thick, and dashed arrows, respectively. The errors indicate uncorrected $1 \sigma$ statistical ones, simply calculated by square root of each 5-min count.

In comparison with these simplified emission profiles, the Gaussian-emission profile is a rather realistic one, defined as

$$
v(t)=\left\{\begin{array}{ll}
C \exp \left(-\left(t-T_{\mathrm{PS}}\right)^{2} / 2 \Delta T^{2}\right) & \text { if } 0 \leq t \leq 2 T_{\mathrm{PS}}, \\
0 & \text { otherwise, }
\end{array},\right.
$$

where $C$ is a normalization factor so that time integral of $v(t)$ over $0-2 T_{\mathrm{PS}}$ is unity, and its centroid and standard deviation $\Delta T$ are set to be $T_{\mathrm{PS}}$ and $0.5 T_{\mathrm{PS}}$, respectively. This is because the light curves of the 4-7 MeV nuclear gamma-ray emission, which obviously reflect ion acceleration at the Sun, are approximately described by a Gaussian function (e.g., Chupp et al. 1990; Watanabe et al. 2003). Furthermore, according to various solar X-ray and gamma-ray observations (e.g., Forrest \& Chupp 1983; Watanabe et al. 2006), the rise and peak times of the 4-7 MeV nuclear gamma-ray light curve are roughly similar to those of the HXR emission.

In this work we treat three neutron emission profiles, since it is natural that ions are accelerated in solar flares at the same time as electrons. However, a recent work performed by Sako et al. (2006) suggests that the acceleration time or trapping time of ions in 2005 September 7 flare may be longer than that of electrons. Thus, it should be noted that we might expect more neutron flux at one $\mathrm{AU}$ than in our investigation.

The ON time windows specified by these three profiles are illustrated in Fig. 3. Here we give a brief explanation of the ON time windows, using 981122 flare (left-upper panel of Fig. 3) as an example. Since the $\delta$-emission supposes instantaneous neutron emission at the Sun, the corresponding ON time window starts at $t_{\mathrm{P}}+1.85 \mathrm{~s}$ and ends at $t_{\mathrm{P}}+667 \mathrm{~s}$, lasting for $665 \mathrm{~s}$, where $1.85 \mathrm{~s}$ and $667 \mathrm{~s}$ are time delays of $10 \mathrm{GeV}$ and $100 \mathrm{MeV}$ neutrons, respectively, with respect to photons. Since this flare has $T_{\mathrm{PS}}=341 \mathrm{~s}$, the ON time windows lasts from $343 \mathrm{~s}$ to $1008 \mathrm{~s}$ as measured from $t_{\mathrm{S}}$. The $\mathrm{ON}$ time windows specified by the other two models are longer than by the $\delta$-emission, because these assume prolonged neutron emissions. It should be stated that 5-min counts shown in Fig. 3 seem to be highly variable. This is because normal Poisson error is assigned to each count. In order to explain that the variations are not very large, we introduce a correction factor for errors of 5-min counts in Sect. 4.3.

By excluding all count bins in the $\mathrm{ON}$ time window and two adjacent count bins, two time regions are available in each light curve for background estimation. We then determined the background level of the NM counts by fitting a common quadratic curve to these two time windows through a leastsquare method. The calculated quadratic curve in turn enables us to define the background level inside the ON time window. This removes residual temporal trend (such as seen in the 3-h data of Fig. 3), mostly due to solar diurnal variation with a typical amplitude of $\pm 0.3 \%$; this arises from diffusion and convection of Galactic cosmic-rays below several tens $\mathrm{GeV}$ in interplanetary space (e.g., Parker 1965; Jokipii \& Parker 1970). Finally, all counts in the ON time window are summed up (hereafter $N_{\mathrm{ON}}$ ), and all the interpolated background counts in the same ON time window are likewise accumulated (hereafter $N_{\mathrm{BG}}$ ). Then the signal from solar neutrons in the $\mathrm{ON}$ time window, $N_{\mathrm{S}}$, is available by simply subtracting $N_{\mathrm{BG}}$ from $N_{\mathrm{ON}}$. Here the $N_{\mathrm{S}}$ would probably correspond to the "worst" neutron signals since we treat all counts equally in an $\mathrm{ON}$ time window as neutron signals. Practically speaking, more neutron signals could be expected via optimizing an ON time window for any of the emission models. This will be subject of our future work.

\subsection{Significance of the neutron signal}

For the purpose of calculating the statistical significance of $N_{\mathrm{S}}$, we need to estimate its uncertainty $\Delta N_{\mathrm{S}}$ given by $\sqrt{\Delta N_{\mathrm{ON}}^{2}+\Delta N_{\mathrm{BG}}^{2}}$, where $\Delta N_{\mathrm{ON}}$ and $\Delta N_{\mathrm{BG}}$ represent errors associated with $N_{\mathrm{ON}}$ and $N_{\mathrm{BG}}$, respectively. However these quantities do not obey Poisson statistics, since the lead blocks, used to increase the detection efficiency through multiplication of incident neutrons, often lead to multiple counts in the $\mathrm{BF}_{3}$ counter for one incident neutron. This prevents us from estimating, e.g., simply as $\Delta N_{\mathrm{ON}}=\sqrt{N_{\mathrm{ON}}}$. Therefore, using the NM data, let us derive a correction factor $\Gamma(>1)$ that yields $\Delta N_{\mathrm{ON}}=\Gamma \sqrt{N_{\mathrm{ON}}}$.

We first construct occurrence histograms of the 5-min counts each day, excluding those data that were obtained 3 days after the 

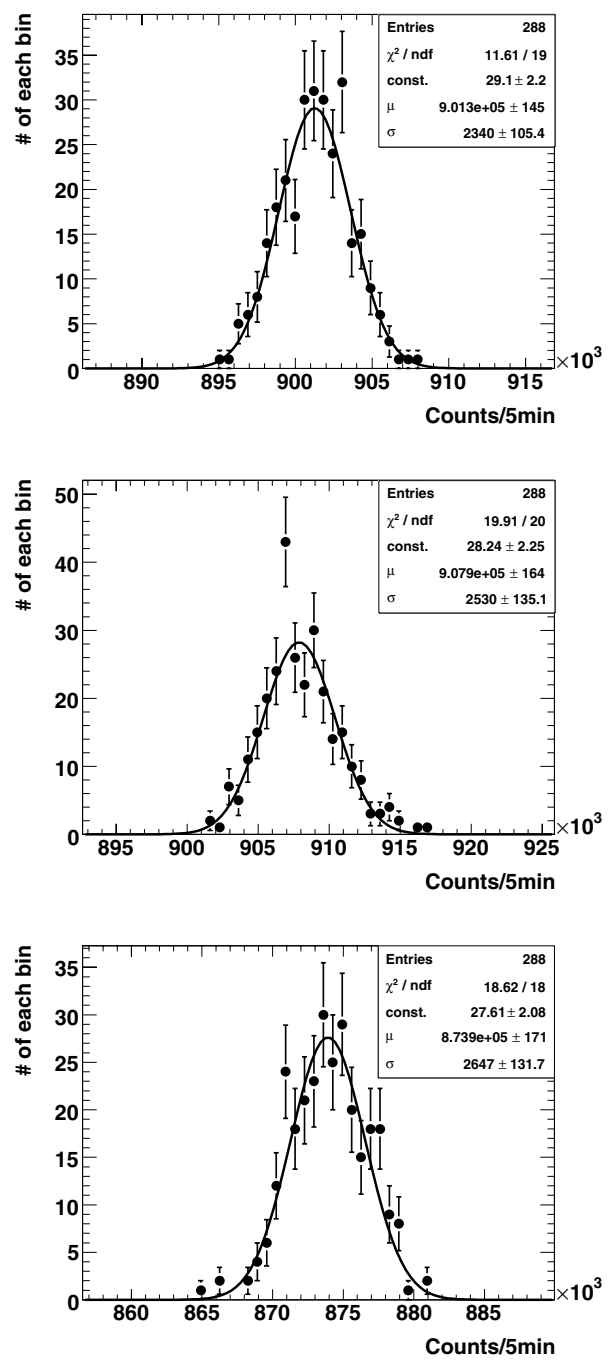

Fig. 4. Typical occurrence histograms of the 5-min count of the Yangbajing NM data on 1998 December 3 (top), 1999 June 3 (middle), and 2000 April 30 (bottom). Error bars assigned to individual data points are Poissonian $\pm 1 \sigma$. The solid line indicates the best Gaussian fit to each distribution.

occurrence of a preliminary-sample flare. This is to avoid possible flare-associated effects, such as the arrival of solar energetic charged particles and/or a subsequent Forbush decrease. The produced histograms are referred to collectively as "backgroundhistogram sample". Then each histogram in the backgroundhistogram sample is fitted with a Gaussian function, in order to derive its mean value, $\mu$, and standard deviation, $\sigma$, and to consequently obtain the factor $\Gamma=\sigma / \sqrt{\mu}$. Here such a multiplicity in NMs was first discussed by Carmichael (1964).

Figure 4 shows some typical distributions in the backgroundhistogram sample. Since the distribution in the top panel of Fig. 4 has $\mu=901270 \pm 145$ and $\sigma=2340 \pm 105$, both per $5 \mathrm{~min}$, we find that $\sigma$ deviates from the expected Poisson fluctuation, $\sqrt{\mu}$, by a factor of $\Gamma=2340 / \sqrt{901270}=2.46 \pm 0.11$. The other distributions shown in Fig. 4 also exhibit similar excess above the expected Poisson fluctuation, yielding $\Gamma=2.65 \pm 0.14$ (middle panel) and $2.83 \pm 0.14$ (bottom panel). Deriving the factor $\Gamma$ from each histogram in the background-histogram sample in the same way, and averaging them over the analyzed period, we obtained the averaged correction factor as $\Gamma=2.73 \pm 0.01$. Furthermore, the standard deviation in 5-min counts has been estimated as $\sigma^{*}=2576 \pm 16$. As can be seen from the particular examples of Fig. 4, the mean $\mu$ varies by $\pm 10-20 \%$ from day to day, due, e.g., to monthly and yearly solar activity changes. The factor $\Gamma$ also varies by a similar extent, depending on the day. However we regard $\Gamma$ as constant, because the fluctuations in $\Gamma$ do not affect our final results. Here, as a general remark, a recent study of NM multiplicity by Bieber et al. (2004) suggests that the multiplicity for a $100 \mathrm{MeV}$ neutron is relatively higher than the derived value in this work. Probably, the difference may result from some systematic differences such as logic of electronics, arrangement of NMs, and geophysical conditions of a station.

Using $\Gamma$ as calculated above, $\Delta N_{\mathrm{ON}}{ }^{2}$ is determined as $\sum\left(\Gamma \sqrt{N_{\mathrm{cnt}}}\right)^{2}=\Gamma^{2} N_{\mathrm{ON}}$, where summation is done over an ON time window, while $\Delta N_{\mathrm{BG}}{ }^{2}$ is evaluated as $\sum\left(\sigma^{*} / \sqrt{n_{\mathrm{off}}}\right)^{2}$, where summation is also performed over the ON time window and $n_{\text {off }}$ is the number of 5-min bins used to define the background level through the least-square method. Finally, the significance of possible neutron signals, $\sigma_{\mathrm{S}}$, can be calculated as

$\sigma_{\mathrm{S}}=\frac{N_{\mathrm{ON}}-N_{\mathrm{BG}}}{\sqrt{\Gamma^{2} N_{\mathrm{ON}}+\sum\left(\sigma^{*} / \sqrt{n_{\mathrm{off}}}\right)^{2}}}$.

\section{Results and discussions}

\subsection{Significance distributions}

With Eq. (2), we calculated the statistical significances of the 18 final-sample flares. Table 2 summarizes the values of $\sigma_{\mathrm{S}}$ for the three neutron production profiles, together with $N_{\mathrm{S}}$ and $\Delta N_{\mathrm{S}}$. Thus, the signal significance is at most $2 \sigma$, implying all null detections. Then, what is the situation for the other flares?

Figure 5 shows occurrence histograms of the statistical significance of signal neutron counts of our preliminary-sample flares, calculated with Eq. (2) in the same way. The 166 flares have been binned into appropriate intervals. We fitted each histogram in Fig. 5 with a Gaussian distribution, with its height, mean value, and standard deviation left free. The fit has been successful as shown in Fig. 5, with two important consequences. One is that the obtained Gaussian centroids are consistent with 0 within the fitting errors. Therefore, there is no evidence in the statistical sense of the positive neutron signals associated with the 166 solar flares. The other point is that the obtained standard deviations are consistent with 1.0, implying that the significance scatter among the 166 flares can be fully explained by statistical fluctuations and that the value of $\Gamma$ has been correctly estimated. This fact also ensures that the developed method is not affected significantly by any unknown systematic effects. For clarifying this, other analyses were performed, systematically including one bin before the beginning of the BATSE start time or leaving out the last bin in ON time windows. The difference between the obtained results shown in Fig. 5 and those other results can be explained by statistical fluctuation.

\subsection{Calculation of an upper limit of the solar-neutron flux}

Given the null detections of solar neutrons, we proceed to set upper limits on the solar-neutron flux from the 18 final-sample flares (Table 1). For this purpose, an upper limit on the neutron counts at $95 \%$ confidence level (CL), $N_{95}$, was estimated for each final-sample flare, from the ON time window counts $N_{\mathrm{S}}$ and its uncertainty $\Delta N_{\mathrm{S}}$, and using a statistical method by Helene (1983). The obtained results on $N_{95}$ are provided in Table 2.

The quantity $N_{95}$ may be written as

$N_{95}=R^{-2} \int_{0}^{\Delta t_{\mathrm{b}}+T_{\max }} \mathrm{d} t \int_{T_{\min }}^{T_{\max }} v\left(t-t^{\prime}\right) N\left(E_{\mathrm{n}}\right) P\left(E_{\mathrm{n}}\right) A\left(\theta, E_{\mathrm{n}}\right) \frac{\mathrm{d} E_{\mathrm{n}}}{\mathrm{d} t^{\prime}} \mathrm{d} t^{\prime}$ 
Table 2. The obtained $N_{\mathrm{S}}, \Delta N_{\mathrm{S}}, N_{95}$, and $\sigma_{\mathrm{S}}$ for each neutron emission profile.

\begin{tabular}{|c|c|c|c|c|c|c|c|c|c|c|c|c|}
\hline \multirow{2}{*}{$\begin{array}{l}\text { Date } \\
\text { (YYMMDD) }\end{array}$} & \multicolumn{4}{|c|}{$\delta$-emission } & \multicolumn{4}{|c|}{ continuous-emission } & \multicolumn{4}{|c|}{ Gaussian-emission } \\
\hline & $N_{\mathrm{S}}$ & $\Delta N_{\mathrm{S}}$ & $N_{95}$ & $\sigma_{\mathrm{S}}$ & $N_{\mathrm{S}}$ & $\Delta N_{\mathrm{S}}$ & $N_{95}$ & $\sigma_{\mathrm{S}}$ & $N_{\mathrm{S}}$ & $\Delta N_{\mathrm{S}}$ & $N_{95}$ & $\sigma_{\mathrm{S}}$ \\
\hline 981112 & -3700 & 3719 & 5263 & -1.0 & -4424 & 4221 & 5874 & -1.1 & -4182 & 4002 & 5572 & -1.1 \\
\hline 981122 & -756 & 3675 & 6724 & -0.2 & -8173 & 6037 & 7644 & -1.4 & -4545 & 5470 & 8152 & -0.8 \\
\hline 981128 & 1418 & 3795 & 8434 & 0.4 & -18620 & 8989 & 9273 & -2.1 & -11409 & 6264 & 6926 & -1.8 \\
\hline 981217 & 3646 & 3532 & 9734 & 1.0 & 4371 & 4175 & 11558 & 1.1 & 3746 & 3886 & 10480 & 1.0 \\
\hline 990402 & 2896 & 3533 & 9098 & 0.8 & 4420 & 4979 & 13105 & 0.9 & 4479 & 4449 & 12159 & 1.0 \\
\hline 990404 & -4626 & 3754 & 4934 & -1.2 & -5345 & 4092 & 5261 & -1.3 & -4776 & 3800 & 4957 & -1.3 \\
\hline 990503 & -1833 & 3603 & 5962 & -0.5 & 4974 & 8601 & 20464 & 0.6 & -243 & 6056 & 11716 & 0.0 \\
\hline 990510 & 656 & 3475 & 7259 & 0.2 & 4582 & 5259 & 13767 & 0.9 & 2229 & 4231 & 9896 & 0.5 \\
\hline 990529 & -1769 & 3458 & 5716 & -0.5 & -6847 & 5862 & 7856 & -1.2 & -2857 & 4197 & 6564 & -0.7 \\
\hline 990604 & -478 & 3488 & 6530 & -0.1 & -1514 & 6251 & 11303 & -0.2 & 2274 & 5583 & 12550 & 0.4 \\
\hline 990724 & -54 & 3495 & 6817 & 0.0 & -3077 & 5892 & 9704 & -0.5 & 881 & 4431 & 9288 & 0.2 \\
\hline 990725 & -608 & 3486 & 6445 & -0.2 & -1479 & 4115 & 7154 & -0.4 & -581 & 3735 & 6950 & -0.2 \\
\hline 991116 & -4199 & 3536 & 4712 & -1.2 & -4866 & 3978 & 5241 & -1.2 & -3539 & 3901 & 5676 & -0.9 \\
\hline 991116 & 1794 & 3735 & 8602 & 0.5 & 1516 & 5040 & 10933 & 0.3 & 2032 & 4780 & 10808 & 0.4 \\
\hline 991126 & -4786 & 3447 & 4322 & -1.4 & -5658 & 5336 & 7396 & -1.1 & -4990 & 4207 & 5609 & -1.2 \\
\hline 000408 & 6955 & 3635 & 12986 & 1.9 & 8412 & 5188 & 17086 & 1.6 & 6986 & 3756 & 13224 & 1.9 \\
\hline 000504 & 3205 & 3589 & 9462 & 0.9 & 2791 & 5628 & 13027 & 0.5 & 2276 & 4694 & 10828 & 0.5 \\
\hline 000515 & -2345 & 3726 & 5927 & -0.6 & 16249 & 9783 & 32585 & 1.7 & 1746 & 6108 & 13183 & 0.3 \\
\hline
\end{tabular}
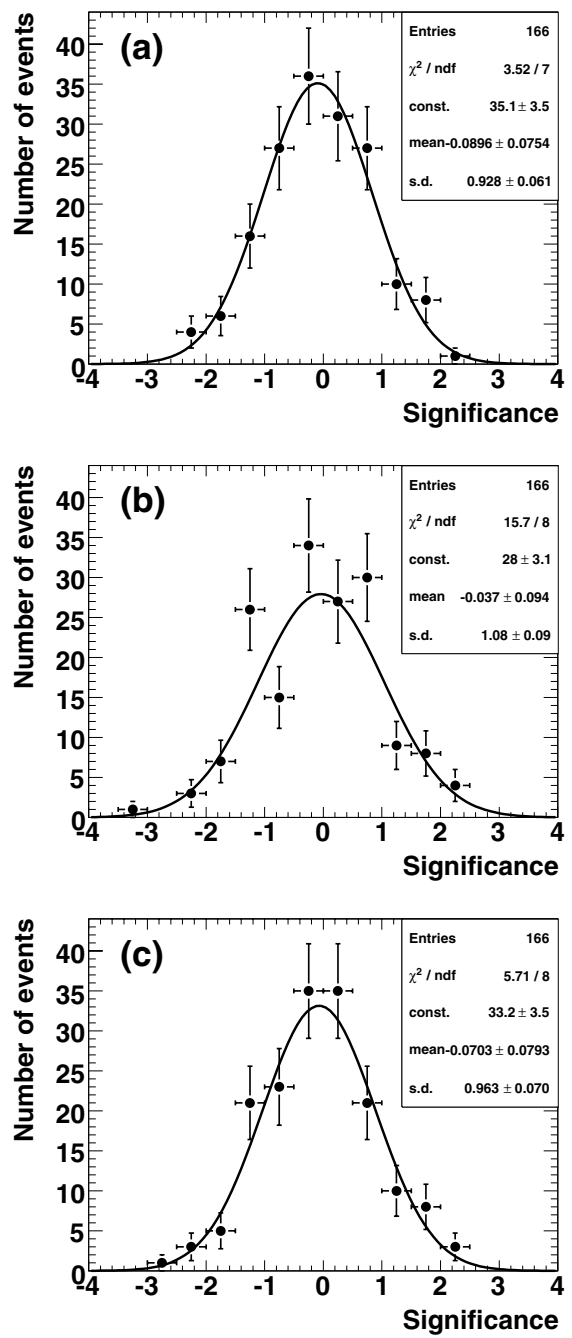

Fig. 5. Significance distributions of neutron signals from our preliminary-sample flares, computed assuming the three neutronemission profiles. Panels a), b), and c) correspond to the $\delta$-, continuous-, and Gaussian-emission models, respectively. Vertical error bars associated with individual data points are Poissonian. The best-fit Gaussian curve to each histogram is drawn by a solid line. Statistical parameters obtained from each fit are indicated in the legend of each graph. where $R$ is the distance from the Sun to the Earth, $t$ the time measured from $t_{\mathrm{S}}, \Delta t_{\mathrm{b}}$ is again the BATSE duration. Also, the times $T_{\min }$ and $T_{\max }$ correspond to delays of $10 \mathrm{GeV}$ and $100 \mathrm{MeV}$ solar neutrons, respectively, the variable $t^{\prime}$ is the difference between the Sun-Earth transit times for neutrons and photons, $E_{\mathrm{n}}$ the kinetic energy of neutrons that is a function of $t^{\prime}$, and $\theta$ the incident angle of neutrons at the top of the atmosphere, which is $\theta_{\text {SUN }}$ in Table 1 . Furthermore, $v$ is the neutron production time profile defined in Sect. 4.2, $N\left(E_{\mathrm{n}}\right)$ the differential neutron spectrum at the Sun, $P\left(E_{\mathrm{n}}\right)$ the probability for a neutron to reach the Earth before its decay, and $A\left(\theta, E_{\mathrm{n}}\right)$ is the effective area of the Yangbajing NM, which is a product of the atmospheric attenuation factor (Fig. 1) and the detection efficiency of an NM64 detector (Clem \& Droman 2000). The term $\mathrm{d} E_{\mathrm{n}} / \mathrm{d} t^{\prime}$ is the neutron energy-time dispersion relation (Lingenfelter \& Ramaty 1967). We assume the function $N\left(E_{\mathrm{n}}\right)$ to have a form of $\alpha\left(E_{\mathrm{n}} / 100 \mathrm{MeV}\right)^{-\beta}\left(\mathrm{sr}^{-1} \mathrm{MeV}^{-1}\right)$, which is theoretically expected from the shock acceleration process of ions in solar flares (see Hua et al. 2002, and references therein). Here, the powerlaw index $\beta$ is assumed to take values of 3,4 , and 5 as conservative ones (Chupp et al. 1987; Muraki et al. 1992; Struminsky et al. 1994; Watanabe et al. 2003, 2006). Since $\theta$ varies from flare to flare (Table 1$), A\left(\theta, E_{\mathrm{n}}\right)$ is interpolated from effective areas calculated for a set of incident angles of $6.6^{\circ}, 10^{\circ}, 20^{\circ}, 30^{\circ}$, $40^{\circ}, 50^{\circ}$, and $60^{\circ}$.

On the right hand side of Eq. (3), the only unknown quantity is the spectrum normalization $\alpha$. Therefore, by substituting the measured values of $N_{95}$ (Table 2) for the left hand side of Eq. (3), we can determine $\alpha$ for each flare. We can then calculate a $95 \%$ CL upper limit on the neutron flux at the top of the Earth atmosphere as

$$
\begin{aligned}
F_{95}= & \alpha \Delta T_{\mathrm{n}}^{-1} R^{-2} \int_{0}^{\Delta t_{\mathrm{b}}+T_{\max }} \mathrm{d} t \\
& \int_{T_{\min }}^{T_{\max }} v\left(t-t^{\prime}\right)\left(E_{\mathrm{n}} / 100 \mathrm{MeV}\right)^{-\beta} P\left(E_{\mathrm{n}}\right) \frac{\mathrm{d} E_{\mathrm{n}}}{\mathrm{d} t^{\prime}} \mathrm{d} t^{\prime},
\end{aligned}
$$

where $\Delta T_{\mathrm{n}}$ is the time interval of the $\mathrm{ON}$ time window. The inner integral of the right hand side of Eq. (4) differs from that of Eq. (3) in that the effective area $A\left(\theta, E_{\mathrm{n}}\right)$ has been eliminated. 

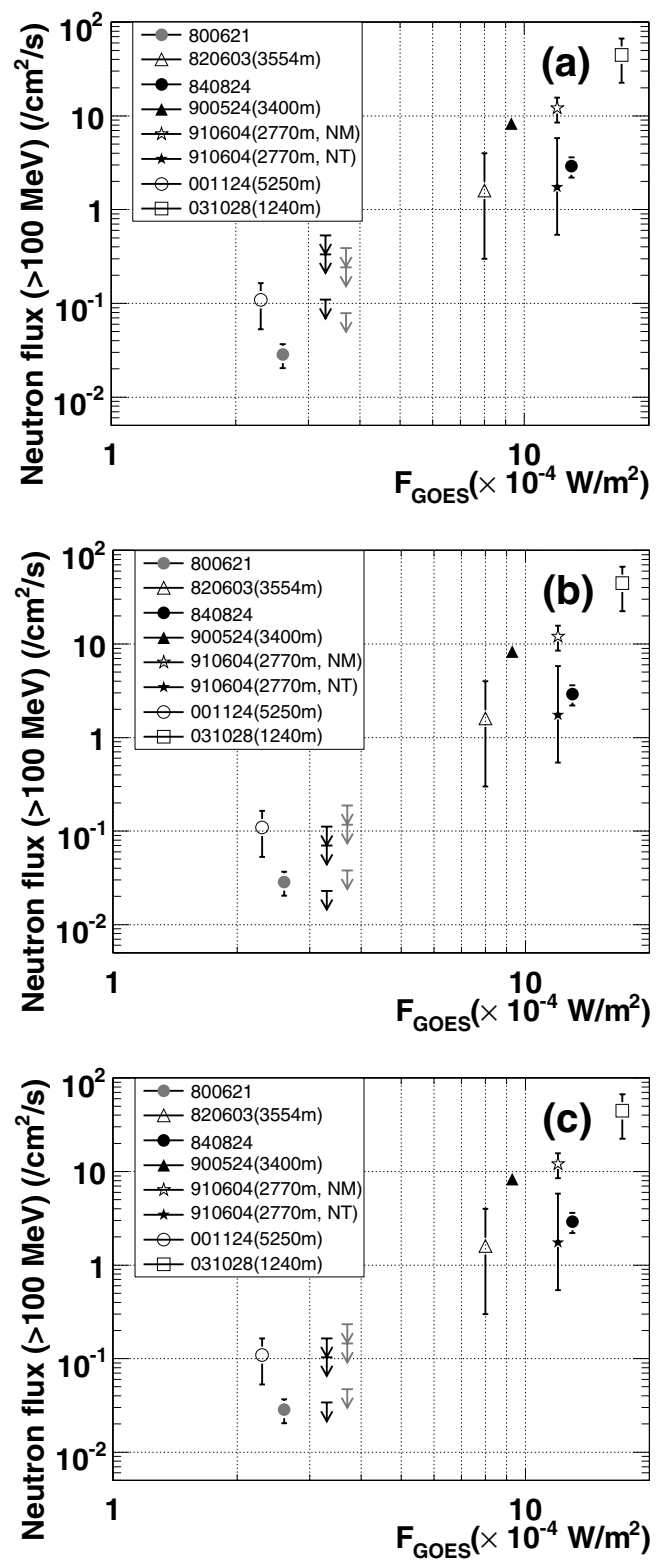

Fig. 6. The $95 \%$ CL upper limits on the $\geq 100 \mathrm{MeV}$ solar-neutron flux at the top of the Earth atmosphere, from 981122 (gray arrows) and 981128 (black arrows) flares, are plotted as a function of $F_{\mathrm{GOES}}$. Previous positive detections are also plotted. Panels a), b), and c) correspond to $\delta$-, continuous-, and Gaussian-emission, respectively. Upper limits from low to high correspond to $\beta$ of 3, 4, and 5, respectively. All errors are only statistical. Except for 800621 (gray filled circle; Ramaty et al. 1983) and 840824 flares (black filled circle; Evenson et al. 1990), observed by satellites, the station altitude is displayed. The references are; Chupp et al. (1987) for the 820603 flare (open triangle), Debrunner et al. $(1993,1997)$ for the 900524 flare (filled triangle), Watanabe et al. (2003) for the 001124 (open circle), and Bieber et al. (2005) and Watanabe et al. (2006) for the 031028 (open square). Neutrons from the 910604 flare were detected by two different detectors installed at Mt. Norikura; the neutron monitor (Struminsky et al. 1994) and the neutron telescope (Muraki et al. 1992).

\subsection{The flux upper limits of the final-sample flares}

Figure 6 and Table 3 show the upper limits on the $\geq 100 \mathrm{MeV}$ solar-neutron flux, $F_{95}$ calculated with Eq. (4), for the two (X3.7 and X3.3) of our final sample flares. For comparison, the previous positive detections (Ramaty et al. 1983; Chupp et al. 1987; Evenson et al. 1990; Muraki et al. 1992; Debrunner et al. 1993;
Table 3. The 95\% CL upper limits on the $\geq 100 \mathrm{MeV}$ solar-neutron flux for two X-class flares.

\begin{tabular}{|c|c|c|c|c|c|c|}
\hline & \multicolumn{3}{|c|}{$\begin{array}{c}981128(\mathrm{X} 3.3) \\
\left(\times 10^{-2} \mathrm{~cm}^{-2} \mathrm{~s}^{-1}\right)\end{array}$} & \multicolumn{3}{|c|}{$\begin{array}{c}981122(\mathrm{X} 3.7) \\
\left(\times 10^{-2} \mathrm{~cm}^{-2} \mathrm{~s}^{-1}\right)\end{array}$} \\
\hline$\beta^{a}$ & $\delta . \mathrm{E}^{b}$ & G.E. ${ }^{c}$ & C.E. ${ }^{d}$ & $\delta$.E. & G.E. & C.E. \\
\hline 3 & 10.9 & 3.4 & 2.3 & 7.9 & 4.7 & 3.8 \\
\hline 4 & 33.4 & 10.3 & 7.0 & 24.2 & 14.5 & 11.7 \\
\hline 5 & 53.2 & 16.5 & 11.2 & 38.9 & 23.3 & 18.8 \\
\hline
\end{tabular}

${ }^{a}$ The power-law index. ${ }^{b} \delta$-emission. ${ }^{c}$ Gaussian emission. ${ }^{d}$ Continuous emission.

Table 4. The most and least stringent upper limits on the $\geq 100 \mathrm{MeV}$ solar-neutron flux.

\begin{tabular}{lccc}
\hline \hline $\begin{array}{l}\text { Date } \\
(\text { YYMMDD })\end{array}$ & Class/optical imp. & $\begin{array}{c}\mathrm{UL}_{\mathrm{M}}^{a} \\
\left(\times 10^{-2} \mathrm{~cm}^{-2} \mathrm{~s}^{-1}\right)\end{array}$ & $\begin{array}{c}\mathrm{UL}_{\mathrm{L}}{ }^{b} \\
\left(\times 10^{-2} \mathrm{~cm}^{-2} \mathrm{~s}^{-1}\right)\end{array}$ \\
\hline 981112 & $\mathrm{M} 1.0 / 1 \mathrm{~N}$ & 4.4 & 23.8 \\
981122 & $\mathrm{X} 3.7 / 1 \mathrm{~N}$ & 3.8 & 38.9 \\
981128 & $\mathrm{X} 3.3 / 3 \mathrm{~N}$ & 2.3 & 53.2 \\
981217 & $\mathrm{M} 3.2 / 1 \mathrm{~N}$ & 22.5 & 97.5 \\
990402 & $\mathrm{M} 1.1 /$ & 3.6 & 22.8 \\
990404 & $\mathrm{M} 5.4 / 1 \mathrm{~F}$ & 1.2 & 7.1 \\
990503 & $\mathrm{M} 4.4 / 2 \mathrm{~N}$ & 0.8 & 5.7 \\
990510 & $\mathrm{M} 2.5 / 2 \mathrm{~N}$ & 1.4 & 6.9 \\
990529 & $\mathrm{M} 1.6 /$ & 1.6 & 14.8 \\
990604 & $\mathrm{M} 3.9 / 2 \mathrm{~B}$ & 0.8 & 6.2 \\
990724 & $\mathrm{M} 3.3 / \mathrm{SF}$ & 1.1 & 9.4 \\
990725 & $\mathrm{M} 1.0 / 1 \mathrm{~F}$ & 1.6 & 9.5 \\
$991116 \mathrm{a}$ & $\mathrm{M} 1.8 / \mathrm{SF}$ & 5.1 & 26.2 \\
$991116 \mathrm{~b}$ & $\mathrm{M} 2.3 / 1 \mathrm{~N}$ & 6.2 & 42.8 \\
991126 & $\mathrm{M} 1.9 / 2 \mathrm{~B}$ & 7.7 & 39.6 \\
000408 & $\mathrm{M} 2.0 / 1 \mathrm{~B}$ & 12.4 & 82.0 \\
000504 & $\mathrm{M} 2.8 / 1 \mathrm{~N}$ & 1.4 & 11.8 \\
000515 & $\mathrm{M} 4.4 /$ & 1.8 & 12.0 \\
\hline
\end{tabular}

${ }^{a}$ The most stringent upper limit. ${ }^{b}$ The least stringent upper limit.

Struminsky et al. 1994; Debrunner et al. 1997; Watanabe et al. 2003; Bieber et al. 2005; Watanabe et al. 2006) are also plotted. Here, the horizontal axis in Fig. 6 gives the GOES class, so the values of 1 and 10 correspond to the GOES class of X1 and X10, respectively. As can be easily seen from Fig. 6, the two present upper limits are comparable to the lowest neutron flux derived from the SMM observation (Chupp et al. 1982) and to the second lowest one from the highest-altitude NM observation (Watanabe et al. 2003). These upper limits, therefore, provide rather stringent constraints on the neutron flux from medium X-class flares, even though they were observed at large zenith angles of $\sim 50^{\circ}$ (Table 1 ).

As shown in Fig. 6, a positive correlation between absolute neutron fluxes and GOES class is expected. The derived upper limits are also consistent with such an expected correlation. Using those absolute fluxes, Fig. 6 reveals a relatively tight and steep dependence of the solar neutron flux on $F_{\mathrm{GOES}}$. In fact, the correlation is quantitatively represented as $F_{\mathrm{n}} \propto F_{\mathrm{GOES}}^{3.0 \pm 0.2}$. (The error of the power-law index is only statistical.) Since the dependence is considerably steeper than direct proportionality, it is strongly suggested that a larger flare not only accelerates a larger number of nucleons, but also accelerates them to higher energies.

The SMM observations of 19 X-class flares, ranging from $\mathrm{X} 1$ to $\mathrm{X} 20$, have provided a sample of $0.8-7 \mathrm{MeV} \mathrm{nu-}$ clear gamma-ray fluxes at the top of the Earth atmosphere 
Table 5. A comparison between upper limits based on two propagation models.

\begin{tabular}{lccccccc}
\hline \hline $\begin{array}{l}\text { emission } \\
\text { profile }\end{array}$ & \multicolumn{3}{c}{$\left.\begin{array}{c}\text { Sh1122 } \\
\left(\times 10^{-2}\right.\end{array} \mathrm{cm}^{-2} \mathrm{~s}^{-1}\right)$} & Ratio $^{a}$ & $\begin{array}{c}\text { Shibata } \\
\left(\times 10^{-2}\right.\end{array}$ & $\begin{array}{c}981128 \\
\left.\mathrm{~cm}^{-2} \mathrm{~s}^{-1}\right)\end{array}$ & Ratio \\
\hline \multirow{3}{*}{$\delta$-emission } & 3 & 2.0 & 7.9 & 0.26 & 2.6 & 10.9 & 0.23 \\
& 4 & 6.5 & 24.2 & 0.27 & 8.1 & 33.4 & 0.24 \\
& 5 & 9.9 & 38.9 & 0.26 & 12.5 & 53.2 & 0.23 \\
\hline \multirow{3}{*}{ gauss-emission } & 3 & 1.2 & 4.7 & 0.26 & 0.8 & 3.4 & 0.23 \\
& 4 & 3.9 & 14.5 & 0.27 & 2.5 & 10.3 & 0.24 \\
& 5 & 6.0 & 23.3 & 0.26 & 3.9 & 16.5 & 0.23 \\
\hline \multirow{2}{*}{ continuous-emission } & 3 & 1.0 & 3.8 & 0.26 & 0.5 & 2.3 & 0.23 \\
& 4 & 3.1 & 11.7 & 0.27 & 1.7 & 7.0 & 0.24 \\
& 5 & 4.8 & 18.8 & 0.26 & 2.6 & 11.2 & 0.23 \\
\hline
\end{tabular}

${ }^{a}$ A ratio of the Shibata model to GHEISHA.

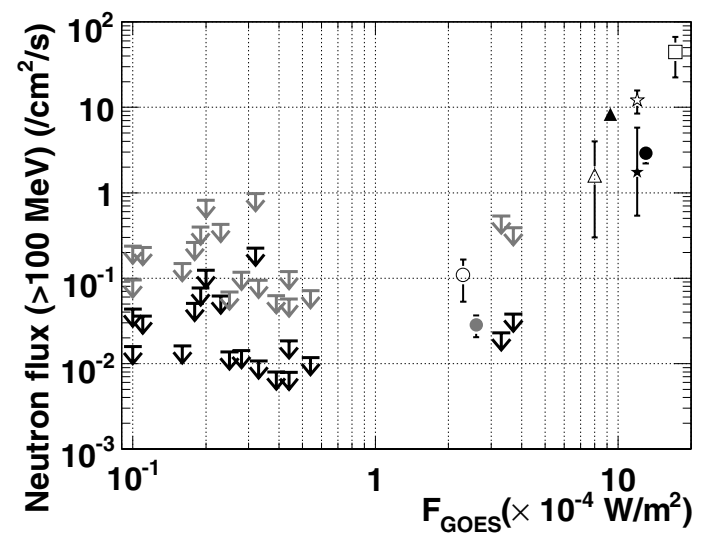

Fig. 7. The same as Fig. 6, but including all the final sample flares. Each black arrow shows the most stringent upper limit for each final sample flare, while the least stringent ones are indicated by gray arrows. The detail information on each data point are shown in the caption of Fig. 6.

(Share \& Murphy 1995). Using the sample, we examined in the same way how the nuclear gamma-ray flux depends on $F_{\mathrm{GOES}}$. As a result, the dependence was found to be much weaker than that of the neutron flux, quantitatively given as $F_{\gamma} \propto F_{\mathrm{GOES}}{ }^{0.32 \pm 0.02}$. (The error is also statistical only.) Thus, the dependence on $F_{\mathrm{GOES}}$ of both secondaries related to ion acceleration is very different, at least, within the quoted statistical errors. The difference between the two relations might be attributed in part to a difference in characteristic ion energies responsible for these emissions: nuclear gamma rays are produced primarily by accelerated ions with energies of $1-100 \mathrm{MeV} / \mathrm{n}$, while $10-100 \mathrm{MeV} / \mathrm{n}$ or even higher energies are necessary for the accelerated ions to efficiently produce high-energy solar neutrons with a detectable flux on the ground (Mandzhavidze \& Ramaty 1993; Aschwanden 2002).

To further examine the relation between the solar-neutron flux and the flare intensity, we show all upper limits from the final sample flares in Fig. 7, and give numerical values of the most and the least stringent upper limits in Table 4. The most stringent upper limits in the present work are obtained assuming that $\beta$ is 3 and $v(t)$ is the continuous-emission, while the least stringent ones are derived assuming that $\beta$ is 5 and $\nu(t)$ is the $\delta$-emission. From the most stringent upper limits, it is found that the neutron flux of M-class flares are probably below $0.01 \mathrm{~cm}^{-2} \mathrm{~s}^{-1}$ at one AU. This is consistent with the fact that neither space observatories nor ground-based detectors have so far succeeded in detecting solar neutrons from M-class solar flares.

\subsection{Dependence on propagation models}

Finally, we examined how our upper limits depend on propagation models used to calculate the atmospheric attenuation factors of solar neutrons. Presently, several kinds of hadronic interaction models are available to deal with complicated nuclear reactions in energies below $10 \mathrm{GeV}$. Among them, the present work has employed GHEISHA in CORSIKA 6.500. However, another model developed by Shibata (1994) has been widely known as one of the representative solar neutron propagation models, because MC simulations based on the Shibata model reproduce experimental results well using $100-400 \mathrm{MeV}$ neutron beams (Koi et al. 2001; Tsuchiya et al. 2001). Thus, a comparison between the GHEISHA and the Shibata models should be carried out.

Using the 981122 (X3.3) and 981128 (X3.7) flares as representative, we computed the flux upper limits using the Shibata model and compared the results with those from GHEISHA in Table 5. Thus the upper limits based on the Shibata model are about a quarter of those based on GHEISHA. Therefore, this factor should be considered as another systematic uncertainty involved in our results. Since every solar neutron observation on the ground is affected by this uncertainty, we must use the same propagation model if trying to compare different observations.

\section{Summary and further prospects}

So far, the solar neutron observations and subsequent analyses have exclusively noticed X-class flares. However, to understand ion acceleration in solar flares, it is very important to examine how the solar neutron flux depends on the flare intensity. For this purpose, we systematically searched the Yangbajing NM data taken from 1998 October to 2000 June for solar neutrons, focusing on 166 BATSE-detected M and X-class flares. No statistically significant signals were found from our small final sample consisting of $2 \mathrm{X}$-class flares and $16 \mathrm{M}$-class ones. Hence, the $95 \%$ confidence level upper limits on the $\geq 100 \mathrm{MeV}$ neutron flux were derived for individual flares. As a consequence, it was found that the upper limits for the two medium X-class flares are comparable to the neutron flux evaluated from the positive detections by the SMM satellite and the highest-altitude NM. These results suggest a very steep dependence of the solar neutron flux on the flare size. Furthermore, we derived useful upper limits on the solar neutron flux from M-class flares for the first time.

Some experiments with newly developed, highly sensitive detectors have been proposed for space missions (Imaida et al. 1999; Moser et al. 2005), as well as for the ground-based stations (Sako et al. 2003). However, for the time being, only detectors on very high mountains, like the Yangbajing NM, would have 
the potential to provide detections of, or strict constraints on, the neutron flux from less intense flares, as well as X-class ones. We plan to utilize another flare sample from, e.g., the Yohkoh satellite and to analyze the NM signals by stacking them in reference to the onset times of a large number of flares.

Acknowledgements. The present research is supported in part by the Special Research Project for Basic Science in RIKEN, titled "Investigation of Spontaneously Evolving Systems". We thank the teams of GOES and BATSE experiments for providing the information on solar X-ray emissions. H. T. thanks Dr. K. Watanabe who kindly calculated the attenuation factor of solar neutrons based on the Shibata model.

\section{References}

Apanasenko, A. V., Sukhadolskaya, V. A., Derbina, V. A., et al. 2001, Astropart. Phys., 16, 13

Apparano, M. V. K., Daniel, R. R., \& Vijayalakshmi, B. 1966, J. Geophys. Res., 71,1781

Asakimori, K., Burnett, J., Cherry, M. L., et al. 1998, ApJ, 502, 278

Aschwanden, M. J. 2002, in Particle Acceleration and Kinematics in Solar Flare-A Synthesis of Recent Observations and Theoretical Concepts (Kluwer Academic Publishers), 143

Bieber, J. W., Clem, J. M., Duldig, M. L., et al. 2004, J. Geophys. Res., 109, A12106

Bieber, J. W., Clem, J., Evenson, P., et al. 2005, Geophys. Res. Lett., 32, L03S02

Cassell, R. E., \& Bower, G. (SLAC) 2002, private communication to D. Heck

Carmichael, H. 1964, Cosmic Rays, IQSY Instruction Manual NO. 7, IQSY Secretariat, London

Chupp, E. L. 1990, ApJ, 73, 213

Chupp, E. L., Forrest, D. J., Ryan, J. M., et al. 1982, ApJ, 263, L95

Chupp, E. L., Debrunner, H., Flückiger, E., et al. 1987, ApJ, 318, 913

Clem, J. M., \& Dorman, L. I. 2000, Space Sci. Rev., 93, 335

Daniel, R. R., Joseph, G., Lavakore, P. J., \& Sundervajan, R. 1967, Nature, 213, 21

Daniel, R. R., Gokhale, G. S., Joseph, G., et al. 1969, Sol. Phys., 10, 465

Debrunner, H., Lockwood, J. A., \& Rayn, J. M. 1993, ApJ, 409, 822

Debrunner, H., Lockwood, J. A., Barat, C., et al. 1997, ApJ, 479, 997

Evenson, P., Kroeger, R., \& Reames, D. 1990, ApJS, 73, 273

Fesefeldt, H. 1985, Report PITHA-85/02, RWTH Aachen

Forrest, D. J., \& Chupp, E. L. 1969, Sol. Phys., 6, 339

Forrest, D. J., \& Chupp, E. L. 1983, Nature, 305, 291
Heck, D., Knapp, J., Capdevielle, J. N., et al. 1998, Report FZKA 6019, Forschungszentrum Karlsruhe

Helene, O. 1983, Nucl. Inst. Meth., 212, 319

Hess, W. N. 1963, Neutrons in Space, Proc. of the Fifth Inter-American Seminar on Cosmic Rays (LaPaz), 17

Hess, W. N., \& Kaifer, R. C. 1967, Sol. Phys., 2, 202

Hua, X. M., Kozlovsky, B., Lingenfelter, R. E., et al. 2002, ApJS, 140, 563

Imaida, I., Muraki, Y., Matsubara, Y., et al. 1999, Nucl. Inst. Meth. A, 421, 99

Jokipii, J. R., \& Parker, E. N. 1970, ApJ, 160, 735

Kasahara, K. 2003, http://eweb.b6.kanagawa-u.ac.jp/ kasahara/ ResearchHome/cosmosHome/index.html

Kohno, T., Miyasaka, H., Matsuoka, M., et al. 1999, Proc. 26th ICRC (Salt Lake City), 6, 62

Koi, T., Muraki, Y., Masuda, K., et al. 2001, Nucl. Inst. Meth. A, 469, 63

Lingenfelter, R. E. 1969, Sol. Phys., 8, 341

Lingenfelter, R. E., \& Ramaty, R. 1967, in High-Energy Nuclear Physics in Astrophysics, ed. W. Shen (Newyork: Benjamin), 99

Lingenfelter, R. E., Flamm, E. J., Canfield, E. H., \& Kellman, S. 1965a, J. Geophys. Res., 70, 4077

Lingenfelter, R. E., Flamm, E. J., Canfield, E. H., \& Kellman, S. 1965b, J. Geophys. Res., 70, 4087

Mandzhavidze, N., \& Ramaty, R. 1993, Nucl. Phys. B 33A,B, 141

Miyasaka, M., Shimoda, S., Yamada, Y., et al. 2001, Proc. 27th ICRC (Hamburg), 3050

Moser, M. R., Flückiger, E. O., Ryan, J. M., et al. 2005, Adv. Space Res., 36, 1399

Muraki, Y., Murakami, K., Miyazaki, M., et al. 1992, ApJ, 400, L75

Parker, E. N. 1965, Planet. Space Sci., 13, 9

Ramaty, R., Murphy, R. J., Kozlovsky, B., \& Lingenfelter, R. E. 1983, ApJ, 273, L41

Sako, T., Muraki, Y., Hirano, N., \& Tsuchiya, H. 2003, Proc. 28th Int. Cosmic Ray Conf. (Tsukuba), 6, 3437

Sako, T., Watanabe, K., Muraki, Y., et al. 2006, ApJ, 651, L69

Sanuki, T., Motoki, M., Matsumoto, H., et al. 2000, ApJ, 545, 1135

Sanuki, T., Matsumoto, H., Nozaki, M., et al. 2001, Adv. Space Res., 27, 761

Share, G. H., \& Murphy, R. J. 1995, ApJ, 452, 933

Shibata, S. 1994, J. Geophys. Res., 99, 6651

Shibata, S., Munakata, Y., Tatsuoka, R., et al. 2001, Nucl. Inst. Meth. A, 463, 316

Stoker, P. H., Dorman, L. I., \& Clem, J. M. 2000, Space Sci. Rev., 93, 361

Struminsky, A., Matsuoka, M., \& Takahashi, K. 1994, ApJ, 429, 400

Tsuchiya, H., Muraki, Y., Masuda, K., et al. 2001, Nucl. Inst. Meth. A, 463, 183

Watanabe, K., Muraki, Y., Matsubara, Y., et al. 2003, ApJ, 592, 590

Watanabe, K., Gros, M., Stoker, P. H., et al. 2006, ApJ, 636, 1135 